\title{
Topographic subtypes of tardive dyskinesia in schizophrenic patients aged less than 60 years: relationship to demographic, clinical, treatment, and neuropsychological variables
}

\author{
O GUREJE
}

\begin{abstract}
From the ARO Neuropsychiatric Hospital, and WHO Collaborating Centre for Research and Training in Mental Health, Abeokuta, Nigeria
\end{abstract}

SUMMARY There are conflicting reports about factors that may be associated with the development of involuntary movements in patients exposed to long-term neuroleptic treatment. Recent evidence suggests that some of these inconsistencies may relate to the practice of regarding these disorders as a unitary syndrome. There is also evidence that both the topographic distribution of these involuntary movements and the pathophysiological mechanisms underlying them may differ in the young and in the old. In this study, demographic, clinical, treatment and neuropsychological variables were investigated for association with the presence of orofacial and limb-truncal dyskinesias in 57 schizophrenic patients aged less than 60 years. Stepwise multiple logistic regression analysis showed that orofacial dyskinesia, at different degrees of severity, was negatively associated with positive schizophrenic syndrome and with either age at assessment or age at onset of illness. None of the indices of schizophrenic deficit (cognitive impairment, clinical defect state, behavioural deterioration, or neurological dysfunction) was related to orofacial dyskinesia. Limb-truncal involuntary movements were negatively related to current daily dosage of neuroleptics. The findings indicate that these two types of dyskinesia may involve diffferent underlying mechanisms and that, for young schizophrenic patients, current pathophysiological or neurochemical theories of involuntary movement disorders are inadequate in explaining their origins.

Recent evidence suggests that the relationship between tardive dyskinesia and long-term treatment with neuroleptic drugs may not be a simple one. Even though these abnormal involuntary movements have long been regarded as a serious and complicating side effect of neuroleptic treatment, ${ }^{12}$ more recent studies have shown that this relationship may not be a direct one. An authoritative review of 56 published studies ${ }^{3}$ shows that the prevalence of tardive dyskinesia ranged from $0.5 \%$ to $56 \%$ (mean, $20 \cdot 1 \%$ ). Thus, the majority of patients treated with neuroleptics do not develop tardive dyskinesia. Also, there are conflicting reports with regard to the associations between tardive dyskinesia and duration of treatment ${ }^{3-5}$ and particular

Address for reprint requests: Dr Oye Gureje, Department of Psychiatry, University Hospital of South Manchester, West Didsbury, Manchester M20 8LR, UK.

Received 15 January 1988 and in revised form 16 June 1988. Accepted 28 July 1988 types of neuroleptics. ${ }^{56}$ Even though many studies have shown an association between these involuntary movements and age, ${ }^{7-9} \mathrm{a}$ few have failed to find such a relationship. ${ }^{4}$

Attempts to find neuropsychological vulnerability factors have produced no consistent results either. There is no consistent result with regard to the associations of tardive dyskinesia with pre-existing brain damage, ${ }^{10-12}$ abnormalities on computed tomography (CT), ${ }^{13-15}$ cognitive dysfunction ${ }^{1316}$ or clinical defect state. ${ }^{17}{ }^{18}$ Recent studies suggesting an excess of non-dextrals ${ }^{19}$ and of soft neurologic signs ${ }^{20}$ among patients with involuntary movements remain to be confirmed.

Various factors may be responsible for these conflicting results. One factor may be the fact that most of the previous studies have regarded these involuntary movements as constituting a unitary syndrome. However, recent evidence suggests that orofacial involuntary movements may be pathophysiologically 
distinct from limb-truncal movements. ${ }^{21}{ }^{22}$ On the basis of blind symptoms ratings, one report ${ }^{23}$ suggests that dyskinetic movements can be grouped into two subsyndromes on principal component analysis: (1) head and neck movements, and (2) trunk and limb movements. Other studies ${ }^{21}$ have noted differential drug response between orofacial and limb and trunk movements. Another factor may relate to age of the patients. Age is particularly important to consider in studies of risk factors for involuntary movements because the prevalence of these movements increases with age ${ }^{8}$ and the topographic distribution of involuntary movements in the young seems different from that in older patients. ${ }^{24}$ Age may also affect the pharmacokinetics of neuroleptics. ${ }^{25}$ A recent comprehensive review of the literature in this area ${ }^{26}$ has shown that differences in the mean age of the study populations may account for some of the inconsistencies noted in studies of patients with tardive dyskinesia with regard to ventricular enlargement, clinical deficits, and cognitive dysfunction. It seems plausible therefore that these movements may result from different pathophysiology in the young from that in the old.

Little is known about the pathophysiology of neuroleptic-induced tardive dyskinesia. Recent observations suggest that the traditional theory of striatal post-synaptic dopamine receptor supersensitivity following prolonged chemical denervation by dopamine blocking antipsychotic drugs is insufficient. There is evidence implicating a noradrenergic hyperactivity. Studies of patients with both tardive dyskinesia and schizophrenia suggest that plasma dopamine- $\beta$ hydroxylase ${ }^{27}$ and cerebrospinal fluid norepinephrine level ${ }^{28}$ are increased compared with schizophrenic patients without tardive dyskinesia.

A number of recent reports have sought to explain the occurrence of involuntary movements in patients with schizophrenia in terms of biologic subtypes of this disorder. In particular, some studies ${ }^{15} 182930$ have suggested that these movements, especially those of orofacial structures, are a component of the Type II subtype of the illness, characterised by negative symptoms of affective flattening and poverty of speech. These studies were conducted among predominantly elderly schizophrenic patients and their results suggest that, at least among these groups of elderly patients, the basic pathophysiology underlying these movements relates to structural brain changes.

The present study was an attempt to determine which demographic, treatment, clinical and neuropsychological variables are associated with the presence of involuntary movement disorders in neuroleptictreated schizophrenic patients who were aged less than 60 years. These movements were considered as consisting of two distinct subsyndromes, orofacial and limbtruncal, and the presence of one was regarded as independent of the presence or absence of the other.

\section{Method}

The entire 147 patients resident in the nine wards of the Lantoro (Long-Stay) Annexe of the Aro Neuropsychiatric Hospital, Abeokuta, Nigeria, were studied. Patients were evaluated for the presence of involuntary movements by the author using the Abnormal Involuntary Movement Scale (AIMS). ${ }^{31}$ The evaluation was conducted blind to each patient's clinical and treatment history. An independent psychiatrist, using the same scale, also rated all the patients for the presence of abnormal involuntary movements. Interrater agreement on the presence of abnormal movements at criterion 2 on the AIMS was high $(k=0.92, z=8.56, p<$ 0.0001 ). The criteria of Schooler and $\mathrm{Kane},{ }^{32}$ as adapted by Waddington $e t$ al, ${ }^{29}$ were also applied in order to define a severe form of orofacial dyskinesia: for this definition, a score of 2 in at least two orofacial areas or of 3 in one area was required.

The patients were also given a battery of neuropsychological tests. Cognitive functioning was tested by administering the subtests of orientation, attention, and forward and backward digit spans of the tests of the sensorium as described by Withers and Hinton. ${ }^{33}$ These subtests were chosen because they are more easily given in the local language, Yoruba, which is the first language of the author and the patients and because they are less affected by level of previous education. The motor and sensory lateralisation of the patients was determined by asking them to perform the following tests: for handedness (erase a pencil mark, throw a ball, touch a point on the wall, cut paper with scissors, and mix water with a spoon); for footedness (kick a ball, step on a rubber bug, and touch a point on a wall); for eye dominance (look through a keyhole and point to examiner's nose while closing one eye). Objects needed for the tests were always presented in the midline. Writing was not employed because many of the patients were illiterate. A right hand/foot/eye preference was scored 0 while a left-side preference was scored 1. A total score of sensorimotor laterality was the sum of each patient's scores on all the tests. Two neurologic soft signs chosen because they were unlikely to be affected by dyskinetic movements were assessed. These were right-left confusion and extinction of double simultaneous stimulation (the face-hand test). The assessment was done as suggested by Quitkin et al..$^{34} \mathrm{~A}$ positive sign was scored 1 while negative or equivocal signs were scored 0 . For each patient a sum of scores on both tests was generated.

The mental state of each patient was assessed using the rating scale devised by Krawiecka et al. ${ }^{35}$. In the present study, flattening and incongruity of affect were rated separately. A score of "positive" syndrome was generated from the scores on delusions, hallucinations, incoherence of speech and incongruity of affect while that of "negative" syndrome was the sum of scores on flattening of affect and poverty of speech/muteness. ${ }^{36}$ The current ward behaviour of each patient was also rated using the scale devised by Wing. ${ }^{37}$ This rating was done by trained psychiatric nurses in charge of each ward.

After all the examination of the patients had been completed, the criteria of Feighner et $a^{\beta 8}$ for schizophrenia were applied to their casenotes. Of the 147 patients examined, 70 satisfied these criteria. The following demographic details were obtained from the casenotes: sex, age, age at onset of illness, duration of illness, age at first hospitalisation, number 
of hospitalisations, and duration of current hospitalisation. Detailed treatment histories were also obtained from the casenotes. This was made possible by many years of meticulous record keeping by the medical and nursing staff of the hospital. Virtually all the patients had received orthodox medical care in this same hospital. Information obtained included the following: cumulative length of neuroleptic exposure, of treatment with high-potency neuroleptics, with intramuscular depot neuroleptics, and with antiparkinsonian drugs, exposure to polypharmacy, number of electroconvulsive treatments received, and daily dosage of neuroleptics on day of evaluation for involuntary movements (converted to chlorpromazine equivalents). ${ }^{39}$

A final selection of patients for the present report was made after all the casenotes had been analysed. Fifty seven of the 70 schizophrenic patients were selected on the basis of being less than 60 years of age and having complete data both from the neuropsychological evaluations and casenote information. There were 45 male and 12 female subjects. Their characteristics are shown in table 1.

Three types of dependent variables were generated: (1) the presence or absence of involuntary movements at criterion 2 (mild) on the AIMS on any one orofacial area, (2) the presence or absence of involuntary movements at criterion 2 on at least two orofacial areas or at criterion 3 (moderate) on any one orofacial area, and (3) the presence or absence of involuntary movements at criterion 2 on any one limbtruncal area. Each of these variables was entered into stepwise multiple logistic regression to determine its relationship with the 21 demographic, treatment and neuropsychological variables. The stepwise model allows a hierarchy

Table 1 Characteristics of the 57 patients (45 males, 12 females)

\begin{tabular}{lrrr}
\hline & Mean & SD & Range \\
\hline Age, yr & $37 \cdot 5$ & $10 \cdot 2$ & $16-58$ \\
Age at onset of illness, yr & $24 \cdot 8$ & $6 \cdot 4$ & $14-40$ \\
Age at first hospitalisation, yr & $28 \cdot 8$ & $8 \cdot 8$ & $14-56$ \\
$\begin{array}{l}\text { Duration of illness, yr } \\
\text { Length of current }\end{array}$ & $12 \cdot 4$ & $7 \cdot 9$ & $1-33$ \\
$\begin{array}{l}\text { hospitalisation, mo } \\
\begin{array}{l}\text { numulative length of } \\
\quad \text { neuroleptic exposure, mo }\end{array}\end{array}$ & $60 \cdot 5$ & $86 \cdot 0$ & $1-396$ \\
$\begin{array}{l}\text { Current daily dose of neuro- } \\
\text { leptic (CPZ-eq), mg }\end{array}$ & $465 \cdot 4$ & $100 \cdot 4$ & $7-576$ \\
\hline
\end{tabular}

of independent variables making a significant contribution to the logistic model to be generated. It also automatically rejects variables that are redundant because they are intercorrelated with others. The adequacy of each final logistic model was confirmed by using the goodness-of-fit tests of Hosmer and Lemeshow ${ }^{40}$ and of Brown." This method of analysis is similar to that used by Waddington et al. ${ }^{29}$

\section{Results}

When the presence of orofacial involuntary movements was determined by a rating on AIMS of 2 on any one orofacial area, the prevalence was $26 \%$ (15/57). For the more stringent case selection of a score of 2 on AIMS on at least two orofacial areas or a score of 3 on any one area, the prevalence was $19 \%$ (11/57). Nine (16\%) of the patients had both types of involuntary movement (using the criterion of a score of 2 or more on AIMS). Table 2 shows the hierarchies of variables that were significantly related by stepwise multiple logistic regression to the three dependent variables as described in the "Method" section.

The variable most significantly related to the presence of the less stringent definition of orofacial involuntary movements was the positive syndrome score. This variable has a negative association with this degree of orofacial dyskinesia. Next to enter the regression was age which was positively related to the presence of this form of motor disorder. When the more stringent definition of orofacial involuntary movements was used, positive syndrome score remained the most strongly associated variable. The association also remained negative. However, for this degree of orofacial motor disorder, age at onset of the schizophrenic illness was the second most strongly associated variable and after which none other entered the regression equation. The association was relatively modest. None of the variables relating to drug treatment bore any relationship to either of the two definitions of orofacial dyskinesia.

The daily dosage of neuroleptics on the day of evaluation for motor disorder was the only variable

Table 2 Variables significantly related by stepwise logistic regression analysis to presence of oro-facial or limb-truncal involuntary movements

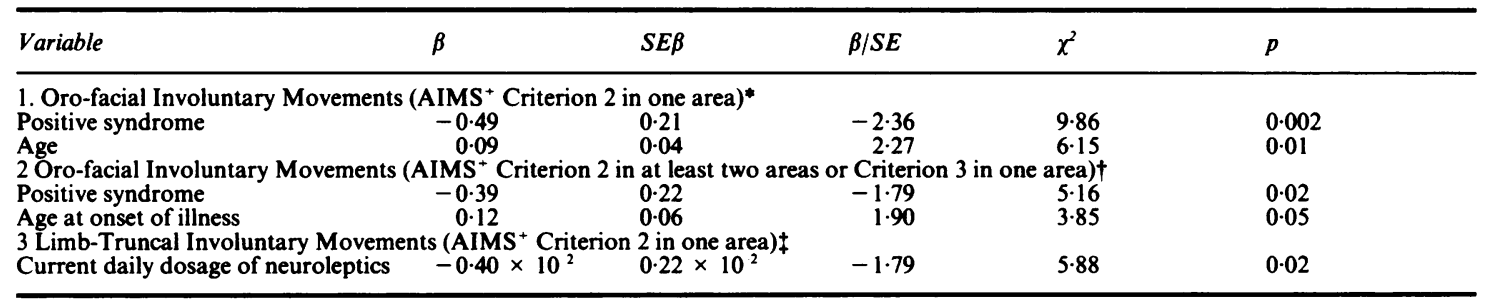

AIMS $^{+}$indicates Abnormal Involuntary Movement Scale.

$*$ Goodness-of-fit tests: Hosmer, $\chi^{2}=11 \cdot 23, p=0 \cdot 13$; Brown, $\chi^{2}=0.24, p=0.89$

+Goodness-of-fit tests: Hosmer, $\chi^{2}=3 \cdot 13, p=0.79$; Brown, $\chi^{2}=0.44, p=0.80$.

$\ddagger$ Goodness-of-fit tests: Hosmer, $\chi^{2}=2 \cdot 88, \mathrm{p}=0.72$; Brown, $\chi^{2}=0.79, \mathrm{p}=0.67$. 
that bore a significant (and negative) association with the presence of limb-truncal involuntary movements. No association was found between limb-truncal dyskinesia and either cumulative length of exposure to neuroleptic or particular types of neuroleptics.

No other demographic, treatment, clinical or neuropsychological variable entered the equation for any of the dependent variables.

\section{Discussion}

Compared with studies ${ }^{46}$ conducted among patients of comparable age, the patients in the present study had received neuroleptics for relatively less cumulative length of time and were currently receiving a lower dosage of neuroleptics. This is in keeping with observations that inpatients in Western countries are frequently given relatively higher doses of neuroleptics. In spite of this, the prevalence of involuntary movements is similar to that frequently reported among patients in Western countries. ${ }^{3}$ Such similarity has been previously noted. ${ }^{42}$ Even though this was a relatively small sample size for examining the issue of prevalence of abnormal involuntary movements, it is interesting to compare the prevalence rates of the topographic subtypes of involuntary movements noted in the present study with those of a recently reported study ${ }^{29}$ with which the present study is identical in respect of case selection and criterion definition. The mean age of the 88 schizophrenic patients who formed the sample of that study was 62.6 years (SD:13.4). For orofacial dyskinesia at criterion 2 on any one area, they observed a prevalence rate of $42 \%$; at criterion 2 on two or more areas the rate was $29 \%$; while for limb-truncal dyskinesia at criterion 2 , the prevalence rate was $14 \%$. It could thus be seen that while orofacial dyskinesia was more prevalent among this elderly group of schizophrenic patients, the reverse was true for limb-truncal involuntary movements which were more prevalent in the present, much younger group of patients with similarly defined illness. This is in keeping with previous observations in respect of the relationship between age and the topographic distribution of these motor disorders. ${ }^{24} 43$

One of the central findings of this study is that different factors are associated with orofacial and limb-truncal involuntary movements. While illness or patient related variables bore significant associations with orofacial dyskinesia, treatment factors seem more important in the pathogenesis of limb-truncal dyskinesia. These findings are in line with those of others $^{29434}$ that involuntary movement disorders affecting predominantly lower-third, midline facial structures may be produced by factors other than treatment. The findings also underscore the observation of previous workers that these topographic subtypes of involuntary movements should be considered separate syndromes and with possibly different underlying pathophysiological mechanisms. ${ }^{21-23}$

The present study was also concerned with the possibility of identifying clinical and neuropsychological measures that could serve as potential vulnerability factors for the development of these subtypes of involuntary movements in a relatively young population. For this reason, various standardised measures of neuropsychological functioning were employed. Indeed, a feature such as sensorimotor laterality, which had hitherto received little attention ${ }^{19}$ in relation to involuntary movement disorders, was assessed in order that variables that might possibly be related to these disorders were not left out. This was especially so since some reports ${ }^{45}$ have suggested the possibility that tardive dyskinesia may be associated with lateralised brain abnormality. In the event, no measure of neuropsychological function was associated with the presence of either oro-facial or limb-truncal involuntary movements.

The results showed that oro-facial involuntary movements were significantly inversely related to positive schizophrenic syndrome (or the Type I syndrome) but not to negative (or Type II) syndrome. It also failed to find an association between such movements and other features that are said to characterise patients with the Type II (or deficit) form of schizophrenia such as cognitive impairment, behavioural deficits ${ }^{153048}$ or increased sinistrality. ${ }^{46}$ These observations are different from those of some previous studies. ${ }^{182943}$ Waddington et al ${ }^{29}$ found that marked cognitive dysfunction or muteness and flattening of affect were significantly related to presence of orofacial dyskinesia in their sample. One feature that is common to all these other studies is the fact that they were conducted among patients who were much older than those in the present study. Studies that have involved a younger patient group have generally found no such relationship between involuntary movements and these measures of schizophrenic deficit. ${ }^{16}{ }^{1747}$ It is interesting to note the same pattern of age difference in studies that have considered the issue of structural brain abnormality in relation to involuntary movements. While studies conducted among elderly schizophrenic patients ${ }^{1315}$ have observed structural brain pathology, those conducted among younger patients ${ }^{478}$ have not. (See review by Wadding$\left.\operatorname{ton}^{26}\right)$. It seems therefore that structural brain pathology, which is said to underlie the Type II schizophrenic syndrome, ${ }^{30}$ is related to involuntary movements especially of the orofacial region in elderly but not in young schizophrenic patients.

The negative association of positive (Type I) syndrome with orofacial dyskinesia in the present study poses a number of difficulties with interpretation. 
First, it could be argued that a negative association with positive syndrome suggests a possible trend which failed to reach statistical significance for orofacial dyskinesia to be positively related to negative syndrome. This would be plausible if, as it is suggested by Andreasen and Olsen, ${ }^{46}$ these two syndromes bore significant negative association with one another. However, in the present study, the two syndromes seem to represent two relatively independent dimensions of pathology ${ }^{30}$ as they bore no significant association with one another (Pearson's $r=-0.05$ ). Also, a negative association of a disorder said to result from postsynaptic dopamine receptor supersensitivity with a syndrome which is thought to result from excessive dopaminergic activity ${ }^{30}$ is paradoxical. However, such observations have been previously reported. ${ }^{4950}$ It is interesting to note that these paradoxical observations were made in patients who were also aged under 60 years. It seems likely that current pathophysiological and neurochemical theories of involuntary movements cannot explain the occurrence of these disorders in young subjects.

Even among these relatively young schizophrenic patients, age emerged as a variable bearing significant association with the presence of a mild form of orofacial dyskinesia. An association between age and involuntary movements is almost consistently reported $^{37}$ and age has often emerged as a highly significant predictor in studies conducted among predominantly young schizophrenic patients in which multivariate statistical analyses were used. ${ }^{69}$

When analysis was carried out for the more stringent criterion for orofacial involuntary movements, a significant negative association with positive syndrome remained the first item to enter the logistic equation. This consistency of association underscores the degree of importance that must attach to whatever pathophysiological or neurochemical process that underlies this relationship. Next to enter the equation for this criterion definition of orofacial movement disorder was age at onset of illness. This is similar to the observation of Waddington and Youssef. ${ }^{31}$ Compared with other degrees of association observed in this study, this particular relationship was modest.

The only variable that bore significant relationship to limb-truncal involuntary movements was a lower dosage of neuroleptics on the day of motor assessment. Even though this association was strong, it could be that dosage of neuroleptics on the day of assessment is only an indicator of a more fundamental relationship between these forms of involuntary movement and neuroleptic exposure. On the other hand, it could be that limb-truncal dyskinesia is an idiosyncratic side effect of neuroleptic treatment which becomes unmasked as the dosage of this type of drug is reduced.
This study has further emphasised and extended previous observations that involuntary movements of the orofacial region constitute a distinct subtype from those of the limbs and trunk. The results of the study show that orofacial dyskinesia in schizophrenic patients aged under 60 years is not associated with measures of schizophrenic deficit but bears a paradoxical relationship to positive psychotic symptoms. This would suggest that the underlying pathophysiological mechanism for this type of dyskinesia in young schizophrenic patients differs from that in elderly schizophrenics. The prevalence of limb-truncal involuntary movements among the patients suggests that these forms of dyskinesia may be more typical of young patients. The relationship between neuroleptic exposure and the development of involuntary movements is probably a complex one and susceptible young individuals may develop these disorders relatively early in their treatment so that duration of exposure to neuroleptics may not be an important risk factor for such groups of patients.

I thank Dr. Edward Ringo for assistance in the determination of inter-rater reliability, the nurses at Lantoro Hospital for enthusiastic support and Mr. Brian Farragher and Mrs. Julie Morris for statistical advice. I am also grateful to the consultants at Aro Hospital for allowing their patients to participate and to Professor David Goldberg for his comments on the manuscript.

\section{References}

1 Faurbye A, Rasch PJ, Peterson BP, Brandborg G, Pakkenberg H. Neurological symptoms in pharmacotherapy of psychosis. Acta Psychiatr Scand 1964;40:10-27.

2 Baldessarini RJ, Cole JO, Davis JM, et al. Task Force Report of the American Psychiatric Association on Late Neurological Effects of Antipsychotic Drugs. Washington DC, American Psychiatric Association, 1980.

3 Kane JM, Smith JM. Tardive dyskinesia: Prevalence and risk factors 1959-1979. Arch Gen Psychiatry 1982;39:473-81.

4 Gardos G, Cole JO, La Brie RA. Drug variables in the etiology of tardive dyskinesia: application of discriminant function analysis. Prog Neuropsychopharmacol 1977;1:147-54.

5 Perris C, Dimitrijevic P, Jacobson L, Paulsson P, Rapp W, Froberg H. Tardive dyskinesia in patients treated with neuroleptics. Br J Psychiatry 1979;135:509-14.

6 Chouinard G, Annable L, Ross-Chouinard A, Nestros JN. Factors related to tardive dyskinesia. Am J Psychiatry 1979;136:79-83.

7 Smith JM, Kucharski LT, Oswald WT, Waterman LJ. A systematic investigation of tardive dyskinesia in inpatients. Am J Psychiatry 1979;136:918-22.

8 Smith JM, Baldessarini RJ. Changes in prevalence, severity, and recovery in tardive dyskinesia with age. Arch Gen Psychiatry 1980;37:1368-73.

9 Mukherjee S, Rosen AM, Cardenas C, Varia V, Olarte S. Tardive dyskinesia in psychiatric outpatients: a study of prevalence and association with demographic, clinical, and drug history variables. Arch Gen Psychiatry 1982;39:466-9. 
10 Edwards $\mathrm{H}$. The significance of brain damage in persistent oral dyskinesia. Br J Psychiatry 1970;116:271-5.

11 Brandon S, McClelland HA, Protheroe C. A study of facial dyskinesia in a metal hospital population. Br J Psychiatry 1971;118:171-84.

12 Jus A, Pineau R, Lachance R, et al. Epidemiology of tardive dyskinesia. Dis Nerv Syst 1976;37:210-4.

13 Famuyiwa OO, Eccleston D, Donaldson AA, Garside RF. Tardive dyskinesia and dementia. $\mathrm{Br} J$ Psychiatry 1979;135:500-4.

14 Jeste DV, Wagner RL, Weinberger DR, Rieth KG, Wyatt RJ. Evaluation of CT scans in tardive dyskinesia. Am J Psychiatry 1980;137:247-8.

15 Owens EGC, Johnstone EC, Crow TJ, Frith CD, Jagoe JR, Kreel L. Lateral ventricular size in schizophrenia: relationship to the disease process and its clinical manifestations. Psychol Med 1985;15:27-41.

16 Wolf ME, Ryan JJ, Mosnaim AD. Cognitive functions in tardive dyskinesia. Psychol Med 1983;13:671-4.

17 Opler LA, Kay SR, Rosado V, Lindenmayer JP. Positive and negative syndromes in chronic schizophrenic inpatients. $J$ Nerv Ment Dis 1984;172:312-5.

18 Waddington JL, Youssef HA. Late onset involuntary movements in chronic schizophrenia: relationship of "tardive" dyskinesia to intellectual impairment and negative symptoms. $B r J P s y$ chiatry 1986;149:616-20.

19 McCreadie RG, Crorie J, Barrom ET, Winslow GS. The Nithsdale schizophrenic survey: III. Handedness and tardive dyskinesia. Br J Psychiatry 1982;140:591-4.

20 Wegner JT, Catalano F, Gilbralter J, Kane JM. Schizophrenics with tardive dyskinesia: neuropsychological deficit and family psychopathology. Arch Gen Psychiatry 1985;42:860-5.

21 Casey DE,Denney D. Pharmacological characterisation of tardive dyskinesia. Psychopharmacol 1977;54:1-9.

22 Barnes TR, Ross M, Trauer T. A comparison of purposeless movements in psychiatric patients treated with antipsychotic drugs, and normal individuals. J Neurol Neurosurg Psychiatry 1983;46:540-6.

23 Kidger T, Barnes TRE, Trauer T, Taylor PJ. Sub-syndromes of tardive dyskinesia. Psychol Med 1980;10:513-20.

24 Tarsy D, Granacher R, Bralower M. Tardive dyskinesia in young adults. Arch Gen Psychiatry 1977;134:1032-4.

25 Yesavage JA, Holman CA, Cohn R, Lombrozo L. Correlation of acute thiothixine serum levels and clinical response: comparison of fluorometric, gas chromatographic, and red blood cell assays. Arch Gen Psychiatry 1983;40:301-4.

26 Waddington JL. Tardive dyskinesia in schizophrenia and other disorders: associations with ageing, cognitive dysfunction and structural brain pathology in relation to neuroleptic exposure. Human Psychopharmacology 1987;2:11-22.

27 Jeste DV, Kleinman JE, Potkin SG, Luchins OJ, Weinberger DR. Ex uno multi: subtyping the schizophrenic syndrome. Biol Psychiatry 1982;17:199-222.

28 Jeste DV, Doogaji DR, Linnoila M. Elevated CSF norepinephrine in tardive dyskinesia. Br J Psychiatry 1984;144:177-80.

29 Waddington JL, Youssef HA, Dolphin C, Kinsella A. Cognitive dysfunction, negative symptoms, and tardive dyskinesia in schizophrenia: their association in relation to topography of involuntary movements and criterion of their abnormality. Arch Gen Psychiatry 1987;44:907-12.
30 Crow TJ. The two-syndrome concept: origins and current status. Schizophr Bull 1985;11:471-86.

31 National Institute of Mental Health: Abnormal Involuntary Movement Scale. In Guy W (ed): Early Clinical Drug Evaluation Unit Assessment Manual. Rockville, Md, US Dept of Health and Human Services, 1976, 534-7.

32 Schooler NR, Kane JM. Research diagnoses for tardive dyskinesia. Arch Gen Psychiatry 1982;39:486-7.

33 Withers E, Hinton J. Three forms of the clinical tests of the sensorium and their reliability. Br J Psychiatry 1971;119:1-8.

34 Quitkin F, Rifkin A, Klein DF. Neurologic soft signs in schizophrenia and character disorders. Arch Gen Psychiatry 1976;33:845-53.

35 Krawiecka M, Goldberg D, Vaughan M. A standardised psychiatric assessment for rating chronic psychotic patients. Acta Psychiatr Scand 1977;55:299-308.

36 Owens DGC, Johnstone EC. The disabilities of chronic schizophrenia: their nature and the factors contributing to their development. Br J Psychiatry 1980;136:384-95.

37 Wing JK. A simple and reliable subclassification of chronic schizophrenia. J Ment Sci 1961;107:862-75.

38 Feighner JP, Robins E,Guze SB, Woodruff RA, Winokur G. Diagnostic criteria for use in psychiatric research. Arch Gen Psychiatry 1972;26:57-63.

39 Davis JM. Comparative doses and costs of antipsychotic medication. Arch Gen Psychiatry 1976;33:858-61.

40 Hosmer D, Lemeshow S. Goodness-of-fit tests for the multiple logistic regression model. Commun Stat Theor Meth 1950;A9:1043-69.

41 Brown CC. A generalisation of the probit and logit methods for dose response curves. Biometrics 1976;32:761-8.

42 Odejide AO. Prevalence of persistent abnormal involuntary movements among patients in a Nigerian long-stay psychiatric unit. Int Pharmacopsychiat 1980;15:292-300.

43 Owens DGC, Johnstone EC, Frith CD. Spontaneous involuntary disorders of movement: Their prevalence, severity, and distribution in chronic schizophrenics with and without treatment with neuroleptics. Arch Gen Psychiatry 1982;39:452-61.

44 Turek IS. Drug-indcued dyskinesia: reality or myth. Dis Nerv Sys 1975;36:397-9.

45 Waziri $R$. Lateralisation of neuroleptic-induced dyskinesia indicates pharmacologic asymmetry of the brain. Psychopharmacol 1980;68:51-3.

46 Andreasen NC, Olsen S. Negative v positive schizophrenia: definition and validation. Arch Gen Psychiatry 1982;39:789-94.

47 Kolakowska T, Williams AO, Ardern M, Reveley MA. Tardive dyskinesia in schizophrenics under 60 years of age. Biol Psychiatry 1986;21:161-9.

48 Brainin M, Reisner T, Zeitlhofer J. Tardive dyskinesia: clinical correlation with computed tomography in patients aged less than 60 years. J Neurol Neurosurg Psychiatry 1983;46:1037-40.

49 Cutler NR, Post RM, Rey AC, Bunney Jr WE. Depressiondependent dyskinesias in two cases of manic-depressive illness. N Engl J Med 1981;304:1088-9.

50 De Potter RW, Linkowski P, Mendlewicz J. State-dependent tardive dyskinesia in manic-depressive illness. $J$ Neurol Neurosurg Psychiatry 1983;46:666-8.

51 Waddington J, Youssef $\mathrm{H}$. Involuntary movements and cognitive dysfunction in late onset schizophrenic outpatients. Irish Med J 1986;79:347-50. 